\title{
Concavity and convexity of several maps involving Tracy-Singh products, Khatri-Rao products, and operator-monotone functions of positive operators
}

\author{
Arnon Ploymukda, Pattrawut Chansangiam* \\ Department of Mathematics, Faculty of Science, King Mongkut's Institute of Technology Ladkrabang, \\ Bangkok 10520 Thailand
}

*Corresponding author, e-mail: pattrawut.ch@kmitl.ac.th

Received 12 Nov 2018

Accepted 27 Mar 2019

\begin{abstract}
We establish concavity and convexity theorems for a number of operator-valued maps involving TracySingh products and Khatri-Rao products of positive operators on a Hilbert space. Operator means serve as useful tools for some convexity results. We also investigate certain maps dealing with positive operator-monotone functions. In this case, the concavity and the convexity of such maps are examined through suitable integral representations of the operator-monotone functions on the unit interval with respect to finite Borel measures.
\end{abstract}

KEYWORDS: positive operator, Tracy-Singh product, Khatri-Rao product, operator-monotone function, Bochner integration

MSC2010: 46G10 47A05 47A63 47A64 47A80

\section{INTRODUCTION}

This paper focuses on concavity and convexity of certain maps dealing with Tracy-Singh products and Khatri-Rao products of operators. Such operator products are generalizations of famous matrix products in the literature, namely, the Kronecker product, the Hadamard product, the Tracy-Singh product, and the Khatri-Rao product.

Recall that the Kronecker product is defined for two matrices $A=\left[a_{i j}\right]$ and $B$ of arbitrary sizes resulting in a block matrix

$$
A * B=\left[a_{i j} B\right]_{i j} .
$$

The Hadamard product is defined for two matrices $A$ and $B$ of the same size

$$
A \circ B=\left[a_{i j} b_{i j}\right] .
$$

Concavity and convexity properties of several matrix-valued maps involving Kronecker products and Hadamard products were collected in Refs. 1-3. As a generalization of the Kronecker product, the Tracy-Singh product ${ }^{4}$ is defined for partitioned matrices $A=\left[A_{i j}\right]$ and $B=\left[B_{k l}\right]$ by

$$
A \circledast B=\left[\left[A_{i j} * B_{k l}\right]_{k l}\right]_{i j} .
$$

The work of Al-Zhour ${ }^{5}$ extends some results of Ando ${ }^{1}$ to Tracy-Singh products of positive definite matrices. The Khatri-Rao product ${ }^{6}$, as a generalized Hadamard product, for $A=\left[A_{i j}\right]$ and $B=\left[B_{i j}\right]$ in the same block-matrix form, is defined by

$$
A \odot B=\left[A_{i j} * B_{i j}\right]_{i j} .
$$

In functional analysis aspect, the tensor product of Hilbert space operators can be viewed as an infinite-dimensional extension of the Kronecker product. Mond and Pečarić ${ }^{7}$ extended the matrix results of Ando $^{1}$ to Hilbert space operators and obtained concavity/convexity theorems associated with positive operator-monotone functions. Ref. 8 extended the notion of tensor product for operators and Tracy-Singh product for matrices to the Tracy-Singh product for Hilbert space operators, and supply its algebraic and order properties. Analytic properties of the Tracy-Singh product were discussed in Ref. 9. Ref. 10 introduced the KhatriRao product of Hilbert space operators and gave a relationship between the Khatri-Rao product and the Tracy-Singh product of two operators via isometric selection operators.

In this study, we investigate concavity and convexity of certain maps related to Tracy-Singh products and Khatri-Rao products of oprators. The main tools we use are operator means and suitable integral representations of certain operator-monotone 
functions. Our results in this paper generalize the results known so far for Tracy-Singh and KhatriRao products of matrices and tensor products of operators. Furthermore, we develop new concavity/convexity theorems.

\section{PRELIMINARIES ON TRACY-SINGH AND KHATRI-RAO PRODUCTS}

Throughout this paper, let $\mathscr{H}, \mathscr{H}^{\prime}, \mathscr{K}$ and $\mathscr{K}^{\prime}$ be complex Hilbert spaces. When $\mathscr{X}$ and $\mathscr{Y}$ are Hilbert spaces, the symbol $\mathbb{B}(\mathscr{X}, \mathscr{Y})$ stands for the algebra of bounded linear operators from $\mathscr{X}$ into $\mathscr{Y}$, and when $\mathscr{X}=\mathscr{Y}$, we write $\mathbb{B}(\mathscr{X})$ instead of $\mathbb{B}(\mathscr{X}, \mathscr{X})$. The cone of positive operators on $\mathscr{H}$ is denoted by $\mathbb{B}(\mathscr{H})^{+}$. For self-adjoint operators $A$ and $B$ on the same space, the situation $A \geqslant B$ means that $A-B$ is positive. Denote the set of all positive invertible operators on $\mathscr{H}$ by $\mathbb{B}(\mathscr{H})^{++}$. If $A \in \mathbb{B}(\mathscr{H})^{++}$, we write $A>0$. The identity operator and the zero operator are denoted by $I$ and 0 , respectively.

To define the Tracy-Singh product and the Khatri-Rao product for operators, we decompose

$$
\begin{array}{ll}
\mathscr{H}=\bigoplus_{j=1}^{n} \mathscr{H}_{j}, & \mathscr{H}^{\prime}=\bigoplus_{i=1}^{m} \mathscr{H}_{i}^{\prime}, \\
\mathscr{K}=\bigoplus_{l=1}^{q} \mathscr{K}_{l}, & \mathscr{K}^{\prime}=\bigoplus_{k=1}^{p} \mathscr{K}_{k}^{\prime},
\end{array}
$$

where all $\mathscr{H}_{j}, \mathscr{H}_{i}^{\prime}, \mathscr{K}_{l}$ and $\mathscr{K}_{k}^{\prime}$ are Hilbert spaces. For each $j$, let $U_{j}: \mathscr{H}_{j} \rightarrow \mathscr{H}$ be the canonical embedding

$$
\left(0, \ldots, 0, x_{j}, 0, \ldots, 0\right) \mapsto x_{j} .
$$

Similarly, for each $l$, let $V_{l}: \mathscr{K}_{l} \rightarrow \mathscr{K}$ be the canonical embedding. For each $i$ and $k$, let $P_{i}: \mathscr{H}^{\prime} \rightarrow \mathscr{H}_{i}^{\prime}$ and $Q_{k}: \mathscr{K}^{\prime} \rightarrow \mathscr{K}_{k}^{\prime}$ be the orthogonal projections. Each $A \in \mathbb{B}\left(\mathscr{H}, \mathscr{H}^{\prime}\right)$ and $B \in \mathbb{B}\left(\mathscr{K}, \mathscr{K}^{\prime}\right)$ can be expressed uniquely as operator matrices

$$
A=\left[A_{i j}\right]_{i, j=1}^{m, n}, \quad B=\left[B_{k l}\right]_{k, l=1}^{p, q},
$$

where $A_{i j}=P_{i} A U_{j} \in \mathbb{B}\left(\mathscr{H}_{j}, \mathscr{H}_{i}^{\prime}\right)$ and $B_{k l}=Q_{k} B V_{l} \in$ $\mathbb{B}\left(\mathscr{K}_{l}, \mathscr{K}_{k}^{\prime}\right)$ for each $i, j, k, l$.

Definition 1 Let $A=\left[A_{i j}\right]_{i, j=1}^{m, n} \in \mathbb{B}\left(\mathscr{H}, \mathscr{H}^{\prime}\right)$ and $B=$ $\left[B_{k l}\right]_{k, l=1}^{p, q} \in \mathbb{B}\left(\mathscr{K}, \mathscr{K}^{\prime}\right)$. We define the Tracy-Singh product of $A$ and $B$ to be the bounded linear operator

$$
\begin{aligned}
& A \otimes B=\left[\left[A_{i j} \otimes B_{k l}\right]_{k l}\right]_{i j}, \\
& A \otimes B: \bigoplus_{j=1}^{n} \bigoplus_{l=1}^{q} \mathscr{H}_{j} \otimes \mathscr{K}_{l} \rightarrow \bigoplus_{i=1}^{m} \bigoplus_{k=1}^{p} \mathscr{H}_{i}^{\prime} \otimes \mathscr{K}_{k}^{\prime} .
\end{aligned}
$$

When $m=p$ and $n=q$, we define the Khatri-Rao product of $A$ and $B$ to be the bounded linear operator

$$
A \boxminus B=\left[A_{i j} \otimes B_{i j}\right]_{i j}: \bigoplus_{i=1}^{n} \mathscr{H}_{i} \otimes \mathscr{K}_{i} \rightarrow \bigoplus_{j=1}^{m} \mathscr{H}_{j}^{\prime} \otimes \mathscr{K}_{j}^{\prime} .
$$

Lemma 1 (Refs. 8, 9) Let $A, B, C, D$ be compatible operators. Then

(i) The map $(A, B) \mapsto A \otimes B$ is bilinear and jointly continuous.

(ii) $(A \otimes B)(C \otimes D)=(A C) \otimes(B D)$.

(iii) If $A$ and $B$ are invertible, then $(A \otimes B)^{-1}=A^{-1} \otimes$ $B^{-1}$.

(iv) If $A$ and $B$ are positive, then $(A \otimes B)^{\alpha}=A^{\alpha} \otimes B^{\alpha}$ for any $\alpha>0$.

(v) If $A \geqslant C \geqslant 0$ and $B \geqslant D \geqslant 0$, then $A \otimes B \geqslant C \otimes D \geqslant$ 0.

(vi) If $A>0$ and $B>0$, then $A \otimes B>0$.

Lemma 2 (Ref. 9) Let $A \in \mathbb{B}(\mathscr{H})$.

(i) If $f$ is an analytic function on a region containing the spectra of $A$ and $I \otimes A$, then $f(I \otimes A)=I \otimes f(A)$.

(ii) If $f$ is an analytic function on a region containing the spectra of $A$ and $A \otimes I$, then $f(A \otimes I)=f(A) \otimes I$.

Lemma 3 (Ref. 10) Let $A \in \mathbb{B}(\mathscr{H})$ and $B \in \mathbb{B}(\mathscr{K})$. If $A \geqslant 0$ and $B \geqslant 0$, then $A \boxminus B \geqslant 0$.

Lemma 4 (Ref. 10) There are isometries $Z_{1}$ and $Z_{2}$ such that

$$
A \boxminus B=Z_{1}^{*}(A \otimes B) Z_{2}
$$

for all $A \in \mathbb{B}\left(\mathscr{H}, \mathscr{H}^{\prime}\right)$ and $B \in \mathbb{B}\left(\mathscr{K}, \mathscr{K}^{\prime}\right)$. For the case $\mathscr{H}=\mathscr{H}^{\prime}$ and $\mathscr{K}=\mathscr{K}^{\prime}$, we have $Z_{1}=Z_{2}:=Z$.

Lemma 5 The Khatri-Rao product of operators is jointly continuous.

Proof: It follows from (1) and the continuity of the Tracy-Singh product (Lemma 1).

For each $i=1, \ldots, k$, let $\mathscr{H}_{i}$ and $\mathscr{H}_{i}^{\prime}$ be Hilbert spaces and decompose

$$
\mathscr{H}_{i}=\bigoplus_{r=1}^{n_{i}} \mathscr{H}_{i, r}, \quad \mathscr{H}_{i}^{\prime}=\bigoplus_{s=1}^{m_{i}} \mathscr{H}_{i, s}^{\prime},
$$

where all $\mathscr{H}_{i, r}$ and $\mathscr{H}_{i, s}^{\prime}$ are Hilbert spaces. For a finite number of operator matrices $A_{i} \in \mathbb{B}\left(\mathscr{H}_{i}, \mathscr{H}_{i}^{\prime}\right)$ for $i=1, \ldots, k$, we use the following notations,

$$
\begin{gathered}
\bigotimes_{i=1}^{k} A_{i}=\left(\left(A_{1} \otimes A_{2}\right) \otimes \cdots \otimes A_{k-1}\right) \otimes A_{k}, \\
\bullet_{i=1}^{k} A_{i}=\left(\left(A_{1} \sqcup A_{2}\right) \square \cdots \boxminus A_{k-1}\right) \sqcup A_{k} .
\end{gathered}
$$


Lemma 6 There are isometries $Z_{1}$ and $Z_{2}$

$$
\bigsqcup_{i=1}^{k} A_{i}=Z_{1}^{*}\left(\bigotimes_{i=1}^{k} A_{i}\right) Z_{2}
$$

for any $A_{i} \in \mathbb{B}\left(\mathscr{H}_{i}, \mathscr{H}_{i}^{\prime}\right), i=1, \ldots, k$. If $\mathscr{H}_{i}$ and $\mathscr{H}_{i}^{\prime}$ are the same space for all $i$, the $Z_{1}=Z_{2}:=Z$.

Proof: We proceed by induction on $k$. If $k=2$, the property (2) is true by Lemma 4. Suppose that there exist isometries $R_{1}$ and $R_{2}$ such that

$$
\bullet_{i=1}^{k-1} A_{i}=R_{1}^{*}\left(\bigotimes_{i=1}^{k-1} A_{i}\right) R_{2} \text {. }
$$

By Lemma 4, there are isometries $S_{1}, S_{2}$ such that

$$
\left(\bigsqcup_{i=1}^{k-1} A_{i}\right) \oslash A_{k}=S_{1}^{*}\left[\left(\bigsqcup_{i=1}^{k-1} A_{i}\right) \otimes A_{k}\right] S_{2} \text {. }
$$

Then

$$
\begin{aligned}
\prod_{i=1}^{k} A_{i} & =\left(\prod_{i=1}^{k-1} A_{i}\right) \bullet A_{k} \\
& =S_{1}^{*}\left[\left(\prod_{i=1}^{k-1} A_{i}\right) \otimes A_{k}\right] S_{2} \\
& =S_{1}^{*}\left[R_{1}^{*}\left(\prod_{i=1}^{k-1} A_{i}\right) R_{2} \otimes A_{k}\right] S_{2} \\
& =S_{1}^{*}\left(R_{1}^{*} \otimes I\right)\left[\left(\prod_{i=1}^{k-1} A_{i}\right) \otimes A_{k}\right]\left(R_{2} \otimes I\right) S_{2} \\
& =\left[\left(R_{1} \otimes I\right) S_{1}\right]^{*}\left(\bigotimes_{i=1}^{k} A_{i}\right)\left(R_{2} \otimes I\right) S_{2} .
\end{aligned}
$$

Set $Z_{1}=\left(R_{1} \otimes I\right) S_{1}$ and $Z_{2}=\left(R_{2} \otimes I\right) S_{2}$. Then $Z_{1}$ and $Z_{2}$ are isometries. When $\mathscr{H}_{i}=\mathscr{H}_{i}^{\prime}$ for all $i=$ $1, \ldots, k$, we have $Z_{1}=Z_{2}$ from the construction.

\section{CONCAVITY AND CONVEXITY}

In this section, we provide concavity and convexity theorems related to Tracy-Singh products of operators. First of all, recall the following terminologies.

Definition 2 A function $f:(0, \infty) \rightarrow(0, \infty)$ is said to be operator-monotone if $f[A] \geqslant f[B]$ whenever $A \geqslant B>0$. Here, $f[A]$ is the (continuous) functional calculus of $f$ defined on the spectrum of $A$.

Definition 3 Let $\mathscr{H}_{1}, \ldots, \mathscr{H}_{k}, \mathscr{K}$ be Hilbert spaces. For each $i=1, \ldots, k$, let $E_{i}$ be a convex subset of $\mathbb{B}\left(\mathscr{H}_{i}\right)$. A function $\phi: E_{1} \times \cdots \times E_{k} \rightarrow \mathbb{B}(\mathscr{K})$ is said to be concave if

$$
\begin{aligned}
& \phi\left((1-t) A_{1}+t B_{1}, \ldots,(1-t) A_{k}+t B_{k}\right) \\
& \quad \leqslant(1-t) \phi\left(A_{1}, \ldots, A_{k}\right)+t \phi\left(B_{1}, \ldots, B_{k}\right)
\end{aligned}
$$

for any $A_{i}, B_{i} \in E_{i}(i=1, \ldots, k)$ and $t \in(0,1)$. A function $\phi$ is convex if $-\phi$ is concave. A map between two convex sets is said to be affine if it preserves convex combinations.

Recall that, for each $t \in(0,1)$, the $t$-weighted harmonic mean and the $t$-weighted geometric mean of $A, B \in \mathbb{B}(\mathscr{H})^{++}$is defined respectively by

$$
\begin{aligned}
& A !_{t} B=\left[(1-t) A^{-1}+t B^{-1}\right]^{-1} \\
& A \#_{t} B=A^{1 / 2}\left(A^{-1 / 2} B A^{-1 / 2}\right)^{t} A^{1 / 2}
\end{aligned}
$$

For arbitrary $A, B \in \mathbb{B}(\mathscr{H})^{+}$, we define the $t$ weighted geometric mean of $A$ and $B$ to be

$$
A \#_{t} B=\lim _{\varepsilon \rightarrow 0^{+}}(A+\varepsilon I) \#_{t}(B+\varepsilon I),
$$

where the limit is taken in the strong-operator topology.

Lemma 7 (Ref. 11) For each $t \in[0,1]$, the map $(A, B) \mapsto A !_{t} B$ is concave on $\mathbb{B}(\mathscr{H})^{++} \times \mathbb{B}(\mathscr{H})^{++}$.

The next lemma gives an integral representation of operator-monotone functions on $(0, \infty)$ in terms of Borel measures on $[0,1]$.

Lemma 8 (Ref. 12) Let $f:(0, \infty) \rightarrow(0, \infty)$ be an operator-monotone function. Then there is a finite Borel measure $\mu$ on $[0,1]$ such that

$$
f(x)=\int_{0}^{1} 1 !_{t} x \mathrm{~d} \mu(t), \quad x>0 .
$$

Theorem 1 Let $f:(0, \infty) \rightarrow(0, \infty)$ be an operatormonotone function. If $\phi_{1}: \mathbb{B}(\mathscr{H})^{++} \rightarrow \mathbb{B}\left(\mathscr{H}^{\prime}\right)^{++}$and $\phi_{2}: \mathbb{B}(\mathscr{K})^{++} \rightarrow \mathbb{B}\left(\mathscr{K}^{\prime}\right)^{++}$are concave maps, then the maps

$$
\begin{aligned}
(A, B) & \mapsto f\left[\phi_{1}(A) \otimes \phi_{2}(B)^{-1}\right] \cdot\left(I \otimes \phi_{2}(B)\right), \\
(A, B) & \mapsto f\left[\phi_{1}(A)^{-1} \otimes \phi_{2}(B)\right] \cdot\left(\phi_{1}(A) \otimes I\right)
\end{aligned}
$$

are concave on $\mathbb{B}(\mathscr{H})^{++} \times \mathbb{B}(\mathscr{K})^{++}$.

Proof: Let $A \in \mathbb{B}(\mathscr{H})^{++}$and $B \in \mathbb{B}(\mathscr{K})^{++}$. Then $\phi_{1}(A)>0$ and $\phi_{2}(B)>0$. Lemma 1 implies that $f\left[\phi_{1}(A) \otimes \phi_{2}(B)^{-1}\right]$ and $f\left[\phi_{1}(A)^{-1} \otimes \phi_{2}(B)\right]$ are well-defined operators. By Lemma 8 , there is a finite Borel measure $\mu$ on $[0,1]$ such that (3) holds. Using Bochner integration, we have

$$
\begin{aligned}
f & {\left[\phi_{1}(A) \otimes \phi_{2}(B)^{-1}\right] \cdot\left(I \otimes \phi_{2}(B)\right) } \\
& =\int_{0}^{1}\left\{(I \otimes I) !_{t}\left(\phi_{1}(A) \otimes \phi_{2}(B)^{-1}\right)\right\}\left(I \otimes \phi_{2}(B)\right) \mathrm{d} \mu(t) .
\end{aligned}
$$


For each $t \in[0,1]$, by Lemma 1 we obtain

$$
\begin{aligned}
&\left\{(I \otimes I) !_{t}\left(\phi_{1}(A) \otimes \phi_{2}(B)^{-1}\right)\right\} \cdot\left(I \otimes \phi_{2}(B)\right) \\
&= {\left[(1-t)(I \otimes I)+t\left(\phi_{1}(A) \otimes \phi_{2}(B)^{-1}\right)^{-1}\right]^{-1} } \\
& \cdot\left(I \otimes \phi_{2}(B)\right) \\
&= {\left[\left(I \otimes \phi_{2}(B)^{-1}\right)\right.} \\
&\left.\cdot\left\{(1-t) I \otimes I+t \phi_{1}(A)^{-1} \otimes \phi_{2}(B)\right\}\right]^{-1} \\
&= {\left[(1-t)\left(I \otimes \phi_{2}(B)\right)^{-1}+t\left(\phi_{1}(A) \otimes I\right)^{-1}\right]^{-1} } \\
&=\left(I \otimes \phi_{2}(B)\right) !_{t}\left(\phi_{1}(A) \otimes I\right) .
\end{aligned}
$$

Since the weighted harmonic mean is concave (Lemma 7), so is the map

$(A, B) \mapsto\left\{(I \otimes I) !_{t}\left(\phi_{1}(A) \otimes \phi_{2}(B)^{-1}\right)\right\} \cdot\left(I \otimes \phi_{2}(B)\right)$.

Thus the map (4) is concave. Similarly, the map (5) is concave.

Remark 1 Since $\phi_{1}(A) \otimes \phi_{2}(B)^{-1}$ commutes with $I \otimes \phi_{2}(B)$, we have

$$
\begin{aligned}
f\left[\phi_{1}(A) \otimes \phi_{2}(B)^{-1}\right] \cdot\left(I \otimes \phi_{2}(B)\right) \\
=\left(I \otimes \phi_{2}(B)\right) \cdot f\left[\phi_{1}(A) \otimes \phi_{2}(B)^{-1}\right] .
\end{aligned}
$$

Similarly,

$$
\begin{aligned}
f\left[\phi_{1}(A)^{-1}\right. & \left.\otimes \phi_{2}(A)\right] \cdot\left(\phi_{1}(A) \otimes I\right) \\
& =\left(\phi_{1}(A) \otimes I\right) \cdot f\left[\phi_{1}(A)^{-1} \otimes \phi_{2}(B)\right] .
\end{aligned}
$$

Example 1 Recall that the function $t \mapsto t^{p}$ is operator-monotone for any $0 \leqslant p \leqslant 1$. Given two concave maps $\phi_{1}: \mathbb{B}(\mathscr{H})^{++} \rightarrow \mathbb{B}\left(\mathscr{H}^{\prime}\right)^{++}$and $\phi_{2}: \mathbb{B}(\mathscr{K})^{++} \rightarrow \mathbb{B}\left(\mathscr{K}^{\prime}\right)^{++}$, by Theorem 1 the maps

$$
\begin{aligned}
(A, B) & \mapsto\left[\phi_{1}(A) \otimes \phi_{2}(B)^{-1}\right]^{p} \cdot\left(I \otimes \phi_{2}(B)\right), \\
(A, B) & \mapsto\left[\phi_{1}(A)^{-1} \otimes \phi_{2}(B)\right]^{p} \cdot\left(\phi_{1}(A) \otimes I\right)
\end{aligned}
$$

are concave on $\mathbb{B}(\mathscr{H})^{++} \times \mathbb{B}(\mathscr{K})^{++}$.

Corollary 1 Let $f:(0, \infty) \rightarrow(0, \infty)$ be operatormonotone. If $\phi_{1}: \mathbb{B}(\mathscr{H})^{++} \rightarrow \mathbb{B}\left(\mathscr{H}^{\prime}\right)^{++}$and $\phi_{2}:$ $\mathbb{B}(\mathscr{K})^{++} \rightarrow \mathbb{B}\left(\mathscr{K}^{\prime}\right)^{++}$are concave maps, then the maps

$$
\begin{aligned}
(A, B) & \mapsto f\left[\phi_{1}(A)^{-1} \otimes \phi_{2}(B)\right] \cdot\left(I \otimes \phi_{2}(B)^{-1}\right), \\
(A, B) & \mapsto f\left[\phi_{1}(A) \otimes \phi_{2}(B)^{-1}\right] \cdot\left(\phi_{1}(A)^{-1} \otimes I\right)
\end{aligned}
$$

are convex on $\mathbb{B}(\mathscr{H})^{++} \times \mathbb{B}(\mathscr{K})^{++}$.

Proof: Note that the function $g(x):=f\left(x^{-1}\right)^{-1}$ is operator-monotone. By Lemma 1 , we have

$$
\begin{aligned}
f\left[\phi_{1}(A)^{-1} \otimes \phi_{2}(B)\right]\left(I \otimes \phi_{2}(B)^{-1}\right) \\
\quad=g\left[\phi_{1}(A)^{-1} \otimes \phi_{2}(B)\right]^{-1}\left(I \otimes \phi_{2}(B)^{-1}\right) .
\end{aligned}
$$

Theorem 1 implies the concavity of the map

$$
\begin{aligned}
(A, B) & \mapsto g\left[\phi_{1}(A) \otimes \phi_{2}(B)^{-1}\right] \cdot\left(I \otimes \phi_{2}(B)\right) \\
= & \left\{\left(I \otimes \phi_{2}(B)^{-1}\right) \cdot f\left[\phi_{1}(A)^{-1} \otimes \phi_{2}(B)\right]\right\}^{-1} \\
= & \left\{f\left[\phi_{1}(A)^{-1} \otimes \phi_{2}(B)\right] \cdot\left(I \otimes \phi_{2}(B)^{-1}\right)\right\}^{-1}
\end{aligned}
$$

Thus the map (6) is convex. Similarly, the map (7) is convex.

Theorem 2 Let $f:(0, \infty) \rightarrow(0, \infty)$ be an operatormonotone function. If $\phi_{1}: \mathbb{B}(\mathscr{H})^{++} \rightarrow \mathbb{B}\left(\mathscr{H}^{\prime}\right)^{++}$is a concave map and $\phi_{2}: \mathbb{B}(\mathscr{K})^{++} \rightarrow \mathbb{B}\left(\mathscr{K}^{\prime}\right)^{++}$is an affine map, then the maps

$$
\begin{aligned}
& (A, B) \mapsto f\left[\phi_{1}(A)^{-1} \otimes \phi_{2}(B)\right] \cdot\left(I \otimes \phi_{2}(B)\right), \\
& (A, B) \mapsto f\left[\phi_{2}(B) \otimes \phi_{1}(A)^{-1}\right] \cdot\left(\phi_{2}(B) \otimes I\right)
\end{aligned}
$$

are convex on $\mathbb{B}(\mathscr{H})^{++} \times \mathbb{B}(\mathscr{K})^{++}$.

Proof: By Lemma 8, there is a finite Borel measure $\mu$ on $[0,1]$ such that (3) holds. Then

$$
\begin{aligned}
f & {\left[\phi_{1}(A)^{-1} \otimes \phi_{2}(B)\right] \cdot\left(I \otimes \phi_{2}(B)\right) } \\
& =\int_{0}^{1}\left\{(I \otimes I) !_{t}\left(\phi_{1}(A)^{-1} \otimes \phi_{2}(B)\right)\right\}\left(I \otimes \phi_{2}(B)\right) \mathrm{d} \mu(t) .
\end{aligned}
$$

For each $t \in[0,1]$, it follows from Lemma 1 that

$$
\begin{aligned}
& \left\{(I \otimes I) !_{t}\left(\phi_{1}(A)^{-1} \otimes \phi_{2}(B)\right)\right\} \\
& =\left[(1-t)(I \otimes I)+t\left(\phi_{1}(A)^{-1} \otimes \phi_{2}(B)\right)^{-1}\right]^{-1} \\
& =\left[(1-t)(I \otimes I)+t\left(\phi_{1}(A) \otimes \phi_{2}(B)^{-1}\right)\right]^{-1} \\
& =\left(I \otimes \phi_{2}(B)\right)\left[(1-t)\left(I \otimes \phi_{2}(B)\right)+t\left(\phi_{1}(A) \otimes I\right)\right]^{-1} .
\end{aligned}
$$

The concavity of the map $(A, B) \mapsto(1-t)\left(I \otimes \phi_{2}(B)\right)+$ $t\left(\phi_{1}(A) \otimes I\right)$ and the affinity of the map $(A, B) \mapsto$ $I \otimes \phi_{2}(B)$ together yield the convexity of the map

$$
\begin{aligned}
& (A, B) \mapsto \\
& \left(I \otimes \phi_{2}(B)\right)\left\{(1-t) I \otimes \phi_{2}(B)+t \phi_{1}(A) \otimes I\right\}^{-1}\left(I \otimes \phi_{2}(B)\right) \\
& \quad=\left\{(I \otimes I) !_{t}\left(\phi_{1}(A)^{-1} \otimes \phi_{2}(B)\right)\right\}\left(I \otimes \phi_{2}(B)\right) .
\end{aligned}
$$

Hence the map (8) is convex. Similarly, the map (9) is convex.

\section{Corollary 2 The maps}

$$
\begin{aligned}
(A, B) & \mapsto I \otimes(B \log [B])-\log [A] \otimes B \\
(A, B) & \mapsto(A \log [A]) \otimes I-A \otimes \log [B]
\end{aligned}
$$

are convex on $\mathbb{B}(\mathscr{H})^{++} \times \mathbb{B}(\mathscr{H})^{++}$. 
Proof: Using Lemmas 1 and 2, we obtain

$$
\begin{aligned}
I \otimes(B \log [B]) & -\log [A] \otimes B \\
& =\{I \otimes \log [B]-\log [A] \otimes I\} \cdot(I \otimes B) \\
& =\{\log [I \otimes B]-\log [A \otimes I]\} \cdot(I \otimes B) \\
& =\log \left[(I \otimes B)(A \otimes I)^{-1}\right] \cdot(I \otimes B) \\
& =(I \otimes B) \cdot \log \left[A^{-1} \otimes B\right] .
\end{aligned}
$$

Since $\log x$ is operator-monotone, by Theorem 2 we obtain that the map

$$
(A, B) \mapsto \log \left[A^{-1} \otimes B\right] \cdot(I \otimes B)
$$

is convex. Hence the map (10) is convex. Similarly, the map (11) is convex.

Example 2 Let $\phi_{1}: \mathbb{B}(\mathscr{H})^{++} \rightarrow \mathbb{B}\left(\mathscr{H}^{\prime}\right)^{++}$be a concave map and $\phi_{2}: \mathbb{B}(\mathscr{K})^{++} \rightarrow \mathbb{B}\left(\mathscr{K}^{\prime}\right)^{++}$an affine map. For any $0 \leqslant p \leqslant 1$, we have by Theorem 2 that the maps

$$
\begin{aligned}
& (A, B) \mapsto\left[\phi_{1}(A)^{-1} \otimes \phi_{2}(B)\right]^{p} \cdot\left(I \otimes \phi_{2}(B)\right), \\
& (A, B) \mapsto\left[\phi_{2}(B) \otimes \phi_{1}(A)^{-1}\right]^{p} \cdot\left(\phi_{2}(B) \otimes I\right)
\end{aligned}
$$

are convex on $\mathbb{B}(\mathscr{H})^{++} \times \mathbb{B}(\mathscr{K})^{++}$.

We mention that the maps (5), (7), (9) and (11) are extensions of results discussed in Ref. 7.

\section{CONCAVITY THEOREMS FOR TRACY-SINGH AND KHATRI-RAO PRODUCTS}

In this section, we present concavity theorems for Tracy-Singh products of operators. Concavity theorems for Khatri-Rao products of operators are established by using the concavity theorems for TracySingh products and the connection between the Khatri-Rao and Tracy-Singh products.

The next result generalizes Corollary 6.2 of Ref. 1 to the case of Tracy-Singh product of operators.

Theorem 3 Let $0 \leqslant p_{i} \leqslant 1, i=1, \ldots, k$, be such that $\sum_{i=1}^{k} p_{i} \leqslant 1$. Then the map

$$
\left(A_{1}, \ldots, A_{k}\right) \mapsto \bigotimes_{i=1}^{k} A_{i}^{p_{i}}
$$

is concave on $\mathbb{B}\left(\mathscr{H}_{1}\right)^{++} \times \cdots \times \mathbb{B}\left(\mathscr{H}_{k}\right)^{++}$.

Proof: We proceed by induction on $k$. Clearly, the map $A_{1} \mapsto A_{1}^{p_{1}}$ is concave. Suppose the assertion is generally true for the case $k-1$. If $p_{k}=0$, then the map becomes

$$
\left(A_{1}, \ldots, A_{k}\right) \mapsto\left(\left(A_{1} \otimes A_{2}\right) \otimes \cdots \otimes A_{k-1}\right) \otimes I,
$$

which is concave. If $p_{k}=1$, then $p_{i}=0$ for all $i=$ $1, \ldots, k-1$ and the map is clearly concave. Now suppose $0<p_{k}<1$. By the induction assumption, the map

$$
\phi\left(A_{1}, \ldots, A_{k-1}\right)=\bigotimes_{i=1}^{k-1} A_{i}^{p_{i} /\left(1-p_{k}\right)}
$$

is concave. By applying Theorem 1 with $f(x)=x^{p_{k}}$, the map

$$
\begin{aligned}
& \left(A_{1}, \ldots, A_{k}\right) \mapsto \\
& \quad f\left(\phi\left(A_{1}, \ldots, A_{k-1}\right)^{-1} \otimes A_{k}\right) \cdot\left(\phi\left(A_{1}, \ldots, A_{k-1}\right) \otimes I\right)
\end{aligned}
$$

is concave. We obtain the concavity of the map (12),

$$
\begin{aligned}
f & \left(\phi\left(A_{1}, \ldots, A_{k-1}\right)^{-1} \otimes A_{k}\right)\left(\phi\left(A_{1}, \ldots, A_{k-1}\right) \otimes I\right) \\
& =\left(\phi\left(A_{1}, \ldots, A_{k-1}\right)^{-p_{k}} \otimes A_{k}^{p_{k}}\right)\left(\phi\left(A_{1}, \ldots, A_{k-1}\right) \otimes I\right) \\
& =\phi\left(A_{1}, \ldots, A_{k-1}\right)^{1-p_{k}} \otimes A_{k}^{p_{k}}=\bigotimes_{i=1}^{k} A_{i}^{p_{i}} .
\end{aligned}
$$

A special case of Theorem 3 is when $k=2$.

Corollary 3 For each $r \in(0,1)$, the map

$$
(A, B) \mapsto A^{1-r} \otimes B^{r}
$$

is concave on $\mathbb{B}(\mathscr{H})^{+} \times \mathbb{B}(\mathscr{K})^{+}$.

Proof: Theorem 3 implies that the map (13) is concave on $\mathbb{B}(\mathscr{H})^{++} \times \mathbb{B}(\mathscr{K})^{++}$. Since the TracySingh product is jointly continuous (Lemma 1), this map is also concave on $\mathbb{B}(\mathscr{H})^{+} \times \mathbb{B}(\mathscr{K})^{+}$.

Next, we develop concavity theorems for KhatriRao products of operators.

Theorem 4 Let $0 \leqslant p_{i} \leqslant 1, i=1, \ldots, k$, be such that $\sum_{i=1}^{k} p_{i} \leqslant 1$. Then the map

$$
\left(A_{1}, \ldots, A_{k}\right) \mapsto \bullet_{i=1}^{k} A_{i}^{p_{i}}
$$

is concave on $\mathbb{B}\left(\mathscr{H}_{1}\right)^{++} \times \cdots \times \mathbb{B}\left(\mathscr{H}_{k}\right)^{++}$.

Proof: From Lemma 6, the map $X \mapsto Z^{*} X Z$, taking the Tracy-Singh product $\bigotimes_{i=1}^{k} A_{i}$ into the Khatri-Rao product $\square_{i=1}^{k} A_{i}$, is linear and preserves positivity. Recall that the composition between a linear map and a concave map results in a concave map. Since the map $\left(A_{1}, \ldots, A_{k}\right) \mapsto \bigotimes_{i=1}^{k} A_{i}^{p_{i}}$ is concave by Theorem 3 , we have the concavity of the map is concave. We obtain the concavity of the map from (12), since

$$
\left(A_{1}, \ldots, A_{k}\right) \mapsto Z^{*}\left(\bigotimes_{i=1}^{k} A_{i}^{p_{i}}\right) Z=\bigsqcup_{i=1}^{k} A_{i}^{p_{i}} .
$$


Corollary 4 For each $r \in(0,1)$, the map

$$
(A, B) \mapsto A^{1-r} \boxminus B^{r},
$$

is concave on $\mathbb{B}(\mathscr{H})^{+} \times \mathbb{B}(\mathscr{K})^{+}$.

Proof: It follows from Theorem 4 when $k=2$ together with the continuity of the Khatri-Rao product, Lemma 5.

\section{CONVEXITY THEOREMS FOR TRACY-SINGH AND KHATRI-RAO PRODUCTS}

In this section, we establish convexity theorems for Tracy-Singh products and Khatri-Rao products of operators. Weighted arithmetic/geometric/harmonic means of operators serve as useful tools.

Lemma 9 (Ref. 13) Let $A_{i}, B_{i} \in \mathbb{B}(\mathscr{H})^{+}, 1 \leqslant i \leqslant k$. Then

$$
\left(\bigotimes_{i=1}^{k} A_{i}\right) \#_{t}\left(\bigotimes_{i=1}^{k} B_{i}\right)=\bigotimes_{i=1}^{k}\left(A_{i} \#_{t} B_{i}\right)
$$

Theorem 5 Let $\phi_{i}, i=1, \ldots, k$, be a concave map from $B\left(\mathscr{H}_{i}\right)^{++}$to $B\left(\mathscr{H}_{i}^{\prime}\right)^{++}$. Then the map

$$
\left(A_{1}, \ldots, A_{k}\right) \mapsto \bigotimes_{i=1}^{k} \phi_{i}\left(A_{i}\right)^{-1}
$$

is convex on $\mathbb{B}\left(\mathscr{H}_{1}\right)^{++} \times \cdots \times \mathbb{B}\left(\mathscr{H}_{k}\right)^{++}$.

Proof: Let $t \in[0,1]$. By continuity, we may assume that $A_{i}$ and $B_{i}$ are positive invertible operators. Applying Lemmas 1 and 9 and the arithmeticgeometric means inequality for operators, we have

$$
\begin{aligned}
& \bigotimes_{i=1}^{k} \phi_{i}\left((1-t) A_{i}+t B_{i}\right)^{-1} \\
& \leqslant \bigotimes_{i=1}^{k}\left((1-t) \phi_{i}\left(A_{i}\right)+t \phi_{i}\left(B_{i}\right)\right)^{-1} \\
& \leqslant \bigotimes_{i=1}^{k}\left(\phi_{i}\left(A_{i}\right) \#_{t} \phi_{i}\left(B_{i}\right)\right)^{-1} \\
& =\bigotimes_{i=1}^{k} \phi_{i}\left(A_{i}\right)^{-1} \#_{t} \bigotimes_{i=1}^{k} \phi_{i}\left(B_{i}\right)^{-1} \\
& \leqslant(1-t) \bigotimes_{i=1}^{k} \phi_{i}\left(A_{i}\right)^{-1}+t \bigotimes_{i=1}^{k} \phi_{i}\left(B_{i}\right)^{-1} .
\end{aligned}
$$

Hence the map (15) is convex.

Corollary 5 Let $0<p_{i} \leqslant 1, i=1, \ldots, k$. Then the map

$$
\left(A_{1}, \ldots, A_{k}\right) \mapsto \bigotimes_{i=1}^{k} A_{i}^{-p_{i}}
$$

is convex on $\mathbb{B}\left(\mathscr{H}_{1}\right)^{++} \times \cdots \times \mathbb{B}\left(\mathscr{H}_{k}\right)^{++}$.
Proposition 1 Let $0 \leqslant p_{i} \leqslant 1, i=1, \ldots, k$, and $1 \leqslant q \leqslant 2$ be such that $\sum_{i=1}^{k} p_{i} \leqslant q-1$. Then the map

$$
\left(A_{1}, \ldots, A_{k+1}\right) \mapsto\left(\bigotimes_{i=1}^{k} A_{i}^{-p_{i}}\right) \otimes A_{k+1}^{q}
$$

is convex on $\mathbb{B}\left(\mathscr{H}_{1}\right)^{++} \times \cdots \times \mathbb{B}\left(\mathscr{H}_{k+1}\right)^{++}$.

Proof: By Theorem 3, the map

$$
\left(A_{1}, \ldots, A_{k+1}\right) \mapsto\left(\bigotimes_{i=1}^{k} A_{i}^{p_{i}}\right) \otimes A_{k+1}^{2-q}
$$

is concave on $\mathbb{B}\left(\mathscr{H}_{1}\right)^{++} \times \cdots \times \mathbb{B}\left(\mathscr{H}_{k+1}\right)^{++}$. Clearly, the map

$$
\left(A_{1}, \ldots, A_{k+1}\right) \mapsto\left(\bigotimes_{i=1}^{k} I\right) \otimes A_{k+1}
$$

is affine. It follows from Lemma 1 that the map

$$
\begin{aligned}
& \left(A_{1}, \ldots, A_{k+1}\right) \mapsto \\
& \begin{aligned}
{\left[\left(\bigotimes_{i=1}^{k} I\right) \otimes A_{k+1}\right]\left[\left(\bigotimes_{i=1}^{k} A_{i}^{p_{i}}\right) \otimes A_{k+1}^{2-q}\right]^{-1}\left(\bigotimes_{i=1}^{k} I\right) \otimes A_{k+1} } \\
=\left(\bigotimes_{i=1}^{k} A_{i}^{-p_{i}}\right) \otimes A_{k+1}^{q}
\end{aligned}
\end{aligned}
$$

is convex.

Theorem 6 For each $r \in(0,1)$, the maps

$$
\begin{aligned}
& (A, B) \mapsto A^{-r} \otimes B^{1+r}, \\
& (A, B) \mapsto A^{1+r} \otimes B^{-r}
\end{aligned}
$$

are convex on $\mathbb{B}(\mathscr{H})^{++} \times \mathbb{B}(\mathscr{K})^{++}$.

Proof: The convexity of the map (16) follows from Proposition 1. By continuity, we may assume that $A$ and $B$ are invertible. Lemma 1 implies that

$A^{1+r} \otimes B^{-r}=\left(A^{r} \otimes B^{-r}\right)(A \otimes I)=\left(A \otimes B^{-1}\right)^{r}(A \otimes I)$.

It follows from Lemmas 1 and 8 that

$$
\begin{aligned}
& A^{1+r} \otimes B^{-r}=\int_{0}^{1}\left((I \otimes I) !_{t}\left(A \otimes B^{-1}\right)\right) \mathrm{d} \mu(t)(A \otimes I) \\
& =\int_{0}^{1}\left[(1-t)(I \otimes I)+t\left(A \otimes B^{-1}\right)^{-1}\right]^{-1}(A \otimes I) \mathrm{d} \mu(t) \\
& =\int_{0}^{1}\left[(1-t)(I \otimes I)+t\left(A^{-1} \otimes B\right)\right]^{-1}(A \otimes I) \mathrm{d} \mu(t) \\
& =\int_{0}^{1}(A \otimes I)[(1-t)(A \otimes I)+t(I \otimes B)]^{-1}(A \otimes I) \mathrm{d} \mu(t) .
\end{aligned}
$$


Since the map $A \mapsto A^{-1}$ is convex and the map $(A, B) \mapsto(1-t)(A \otimes I)+t(I \otimes B)$ is affine, the map

$$
(A, B) \mapsto(I \otimes B)\{(1-t)(A \otimes I)+t(I \otimes B)\}^{-1}(I \otimes B)
$$

is convex. Thus the map $(A, B) \mapsto A^{1+r} \otimes B^{-r}$ is convex.

Proposition 2 Let $\phi_{i}, i=1, \ldots, k$, be concave maps from $B\left(\mathscr{H}_{i}\right)^{++}$to $B\left(\mathscr{H}_{i}^{\prime}\right)^{++}$. Then the map

$$
\left(A_{1}, \ldots, A_{k}\right) \mapsto \bullet_{i=1}^{k} \phi_{i}\left(A_{i}\right)^{-1}
$$

is convex on $\mathbb{B}\left(\mathscr{H}_{1}\right)^{++} \times \cdots \times \mathbb{B}\left(\mathscr{H}_{k}\right)^{++}$.

Proof: It follows from Lemma 6 and Theorem 5.

Corollary 6 Let $0<p_{i} \leqslant 1$ for each $i=1, \ldots, k$. Then the map

$$
\left(A_{1}, \ldots, A_{k}\right) \mapsto \bullet_{i=1}^{k} A_{i}^{-p_{i}}
$$

is convex on $\mathbb{B}\left(\mathscr{H}_{1}\right)^{++} \times \cdots \times \mathbb{B}\left(\mathscr{H}_{k}\right)^{++}$.

Proof: It follows from Proposition 2 by putting $\phi_{i}\left(A_{i}\right)=A_{i}^{p_{i}}$ for each $i$.

Proposition 3 For each $r \in(0,1)$, the maps

$$
\begin{aligned}
& (A, B) \mapsto A^{-r} \boxminus B^{1+r}, \\
& (A, B) \mapsto A^{1+r} \boxminus B^{-r}
\end{aligned}
$$

are convex on $\mathbb{B}(\mathscr{H})^{++} \times \mathbb{B}(\mathscr{K})^{++}$.

Proof: It follows from Lemma 4 and Theorem 6.

Recall that the Moore-Penrose inverse of an operator $T \in \mathbb{B}\left(\mathscr{H}, \mathscr{H}^{\prime}\right)$ is the operator $T^{\dagger} \in$ $\mathbb{B}\left(\mathscr{H}^{\prime}, \mathscr{H}\right)$ satisfying the conditions $T T^{\dagger} T=T$, $T^{\dagger} T T^{\dagger}=T,\left(T T^{\dagger}\right)^{*}=T T^{\dagger}$, and $\left(T^{\dagger} T\right)^{*}=T^{\dagger} T$. It is well known that $T^{\dagger}$ exists if and only if the range of $T$ is closed $^{14}$.

Lemma 10 (Ref. 15) Let

$$
T=\left[\begin{array}{ll}
T_{11} & T_{12} \\
T_{12}^{*} & T_{22}
\end{array}\right] \in \mathbb{B}\left(\mathscr{H}_{1} \oplus \mathscr{H}_{2}\right)
$$

be a self-adjoint operator. Suppose that $T_{11}$ has a closed range. Then $T \geqslant 0$ if and only if $T_{11} \geqslant 0$, $T_{12}=T_{11} T_{11}^{\dagger} T_{12}$, and $T_{22} \geqslant T_{12}^{*} T_{11}^{\dagger} T_{12}$.

Recall that for any interval $J$, a continuous function $f: J \rightarrow \mathbb{R}$ is convex if and only if $f(x+$ $h)+f(x-h)-2 f(x) \geqslant 0$ for all $x \in J$ and $h>0$ such that $x \pm h \in J$.
Theorem 7 Let $A \in \mathbb{B}(\mathscr{H})^{+}$and $B \in \mathbb{B}(\mathscr{K})^{+}$have closed ranges. Then the operator-valued function

$$
\begin{aligned}
& \phi:[-1,1] \rightarrow \mathbb{B}\left(\bigoplus_{i=1}^{n} \mathscr{H}_{i} \otimes \mathscr{K}_{i}\right), \\
& \phi(t)=A^{1+t} \backsim B^{1-t}+A^{1-t} \bullet B^{1+t}
\end{aligned}
$$

is convex on $[-1,1]$, decreasing on $[-1,0]$, increasing on $[0,1]$, attains minimality at $t=0$, and attains maximality at $t=-1,1$.

Proof: Let $s \in[-1,1]$ and $t>0$ be such that $s \pm t \in$ $[-1,1]$. Consider the operator matrices

$$
\begin{array}{ll}
T_{1}=\left[\begin{array}{ll}
A^{1+s+t} & A^{1+s} \\
A^{1+s} & A^{1+s-t}
\end{array}\right], & T_{2}=\left[\begin{array}{ll}
A^{1-s-t} & A^{1-s} \\
A^{1-s} & A^{1-s+t}
\end{array}\right], \\
T_{3}=\left[\begin{array}{ll}
B^{1+s+t} & B^{1+s} \\
B^{1+s} & B^{1+s-t}
\end{array}\right], & T_{4}=\left[\begin{array}{ll}
B^{1-s-t} & B^{1-s} \\
B^{1-s} & B^{1-s+t}
\end{array}\right] .
\end{array}
$$

Note that

$$
\begin{aligned}
& A^{1+s}=\left(A A^{\dagger} A\right)^{1+s+t} A^{-t}=A^{1+s+t}\left(A^{1+s+t}\right)^{\dagger} A^{1+s}, \\
& A^{1+s-t}=A^{-t}\left(A A^{\dagger} A\right)^{1+s+t} A^{-t}=A^{1+s}\left(A^{1+s+t}\right)^{\dagger} A^{1+s} .
\end{aligned}
$$

We have by Lemma 10 that $T_{i}$ is positive for all $i=1,2,3,4$. By the monotonicity of Khatri-Rao product, Lemma 3 , we have that the operator $X \equiv$ $T_{1} \square T_{4}+T_{2} \square T_{3}$ is

$\left[\begin{array}{ll}A^{1+s+t} \square B^{1-s-t}+A^{1-s-t} \boxminus B^{1+s+t} & A^{1+s} \square B^{1-s}+A^{1-s} \square B^{1+s} \\ A^{1+s} \square B^{1-s}+A^{1-s} \square B^{1+s} & A^{1+s-t} \square B^{1-s+t}+A^{1-s+t} \backsim B^{1+s-t}\end{array}\right]$,

which is positive. Similarly, the operator $Y$,

$$
\left[\begin{array}{ll}
A^{1+s-t} \boxminus B^{1-s+t}+A^{1-s+t} \bullet B^{1+s-t} & A^{1+s} \bullet B^{1-s}+A^{1-s} \bullet B^{1+s} \\
A^{1+s} \bullet B^{1-s}+A^{1-s} \bullet B^{1+s} & A^{1+s+t} \bullet B^{1-s-t}+A^{1-s+t} \bullet B^{1+s+t}
\end{array}\right]
$$

is also positive. It follows that

$$
\begin{aligned}
0 & \leqslant X+Y=\left[\begin{array}{cc}
\phi(s+t)+\phi(s-t) & 2 \phi(s) \\
2 \phi(s) & \phi(s+t)+\phi(s-t)
\end{array}\right] \\
& =U\left[\begin{array}{cc}
\phi(s+t)+\phi(s-t)+2 \phi(s) & 0 \\
0 & \phi(s+t)+\phi(s-t)-2 \phi(s)
\end{array}\right] U^{*}
\end{aligned}
$$

where

$$
U=\frac{1}{\sqrt{2}}\left[\begin{array}{cc}
I & -I \\
I & I
\end{array}\right] .
$$

Again, Lemma 10 guarantees that

$$
\phi(s+t)+\phi(s-t) \geqslant 2 \phi(s) .
$$

This means that $\phi$ is convex. The fact that $\phi(t)=$ $\phi(-t)$ for all $t \in[-1,1]$ and the convexity of $\phi$ implies that $\phi$ has the minimal value at 0 . Hence $\phi$ is decreasing on $[-1,0]$ and increasing on $[0,1]$. 
Corollary 7 Let $A \in \mathbb{B}(\mathscr{H})^{+}$and $B \in \mathbb{B}(\mathscr{K})^{+}$have closed ranges. Then the parameterization

$$
\begin{aligned}
& \psi:[0,1] \rightarrow \mathbb{B}\left(\bigoplus_{i=1}^{n} \mathscr{H}_{i} \otimes \mathscr{K}_{i}\right), \\
& \psi(t)=A^{t} \boxminus B^{1-t}+A^{1-t} \square B^{t}
\end{aligned}
$$

is convex on $[0,1]$, decreasing on $[0,1 / 2]$, increasing on $[1 / 2,1]$, attains minimality at $t=1 / 2$, and attains maximality at $t=0,1$.

Proof: Let $f:[0,1] \rightarrow[-1,1]$ be defined by $f(t)=$ $2 t-1$. Then $\psi=\phi \circ f$ where $\phi$ is given by (18). Now, the desired results follow from Theorem 7 by using $f([0,1])=[-1,1], f([0,1 / 2])=[-1,0]$, $f([1 / 2,0])=[0,1]$, and $f(1 / 2)=0$.

As a consequence, we obtain an operator version of the arithmetic-geometric mean inequality as follows.

Corollary 8 Let $A \in \mathbb{B}(\mathscr{H})^{+}$and $B \in \mathbb{B}(\mathscr{K})^{+}$have closed ranges. For any $t \in[1 / 2,1]$, we have

$$
2\left(A^{1 / 2} \boxminus B^{1 / 2}\right) \leqslant A^{t} \boxminus B^{1-t}+A^{1-t} \square B^{t} \leqslant A \boxplus B,
$$

where $\boxplus$ denotes the Khatri-Rao sum ${ }^{16}$ defined by $A \boxplus B=A \boxminus I+I \boxminus B$.

We mention that Theorem 5, Corollary 5, and Proposition 1 generalize the matrix results involving Tracy-Singh products provided in Ref. 5.

Acknowledgements: The first author expresses his gratitude towards Thailand Research Fund for providing the Royal Golden Jubilee PhD Scholarship, grant No. PHD60K0225 to support his PhD study.

\section{REFERENCES}

1. Ando T (1979) Concavity of certain maps on positive definite matrices and applications to Hadamard products. Linear Algebra Appl 26, 203-41.

2. Bhatia R (2007) Positive Definite Matrices, Princeton University Press, Princeton, New Jersey.
3. Matharu JS, Aujla JS (2009) Hadamard product versions of the Chebyshev and Kantorovich inequalities. J Inequal Pure Appl Math 10, 1-6.

4. Tracy DS, Singh RP (1972) A new matrix product and its applications in partitioned matrix differentiation. Stat Neerl 26, 143-57.

5. Al-Zhour Z (2014) Several new inequalities on operator means of non-negative maps and Khatri-Rao products of positive definite matrices. $J$ King Saud Univ Sci 26, 21-7.

6. Khatri CG, Rao CR (1968) Solutions to some factional equations and their applications to characterization of probability distributions. Sankhyā 30, 167-80.

7. Mond B, Pečarić JE (1998) Operator convex functions of several variables. Soochow J Math 24, 239-54.

8. Ploymukda A, Chansangiam P, Lewkeeratiyutkul W (2018) Algebraic and order properties of Tracy-Singh products for operator matrices. J Comput Anal Appl 24, 656-64.

9. Ploymukda A, Chansangiam P, Lewkeeratiyutkul W (2018) Analytic properties of Tracy-Singh products for operator matrices. J Comput Anal Appl 24, 665-74.

10. Ploymukda A, Chansangiam P (2016) Khatri-Rao products of operator matrices acting on the direct sum of Hilbert spaces. J Math 2016, 1-7.

11. Hiai F, Petz D (2014) Introduction to Matrix Analysis and Applications, Springer, New Delhi.

12. Chansangiam P, Lewkeeratiyutkul W (2015) Operator connections and Borel measures on the unit interval. ScienceAsia 41, 273-9.

13. Ploymukda A, Chansangiam P (2018) Geometric means and Tracy-Singh products for positive operators. Commun Math Appl 9, 475-88.

14. Caradus SR (1978) Generalized Inverses and Operator Theory, Queen's Papers in Pure and Applied Mathematics 50, Queen's University, Kingston.

15. Xu Q, Sheng L (2008) Positive semi-definite matrices of adjointable operators on Hilbert $C^{*}$-modules. Linear Algebra Appl 428, 992-1000.

16. Ploymukda A, Chansangiam P (2018) Khatri-Rao sums for Hilbert space operators. Songklanakarin $J$ Sci Technol 40, 595-601. 